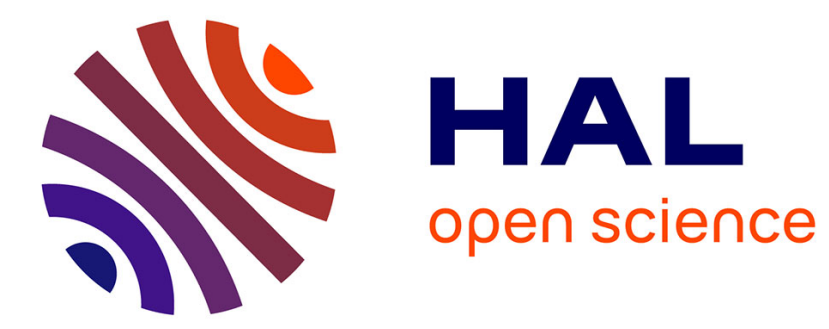

\title{
Medium term load forecasting using ANFIS predictor.
}

Otilia Elena Dragomir, Florin Dragomir, Rafael Gouriveau, Eugénia Minca

\section{To cite this version:}

Otilia Elena Dragomir, Florin Dragomir, Rafael Gouriveau, Eugénia Minca. Medium term load forecasting using ANFIS predictor.. 18th Mediterranean Conference on Control \& Automation, MED'10., Jun 2010, Marrakech, Morocco. pp.551-556. hal-00544696

\section{HAL Id: hal-00544696 \\ https://hal.science/hal-00544696}

Submitted on 8 Dec 2010

HAL is a multi-disciplinary open access archive for the deposit and dissemination of scientific research documents, whether they are published or not. The documents may come from teaching and research institutions in France or abroad, or from public or private research centers.
L'archive ouverte pluridisciplinaire HAL, est destinée au dépôt et à la diffusion de documents scientifiques de niveau recherche, publiés ou non, émanant des établissements d'enseignement et de recherche français ou étrangers, des laboratoires publics ou privés. 


\title{
Medium Term Load Forecasting Using ANFIS Predictor
}

\author{
Otilia Elena DRAGOMIR, Member, IEEE, Florin DRAGOMIR, Rafael GOURIVEAU and Eugenia \\ MINCA, Member, IEEE
}

\begin{abstract}
Nowadays, there are huge ranges of energy market participants. Commercial success of this area actor depends on the ability to submit competitive predictions relative to energy balance trends Thus, it seems convenient to "anticipate" this parameter evolution in time in order to act consequently and resort to protective actions. In this context, this paper proposes a tool for energy balance prediction based on ANFIS (Adaptive Neuro Fuzzy Inference System). This neuro- fuzzy predictor is modified in order to obtain an accurate forecasting for medium term. The solutions are illustrated on a real application and take into account the known "future": the programmed actions.
\end{abstract}

Keywords - ANFIS (Adaptive Neuro Fuzzy Inference System), forecasting, renewable energy, medium term

\section{INTRODUCTION}

C onsidering the benefits that energy forecasting may bring to the security, economics and resource management fields, the scientific community is now beginning to take some interest in this area. The control of the performance prediction represents the premise of a good global performance. The power energy market it is very interested in forecasting energy balance (difference between the electricity produced and consumed - DPcg). Nowadays, there are huge ranges of energy market participants, bidding strategies are more and more complex, and a number of financial derivatives have been developed. Commercial success of this area actor depends on the ability to submit competitive predictions relative to DPcg trends [1].

In the forecasting literature, the term "forecasting" is called also prediction or prognosis. This reveals that there is no consensual acceptation of term: [2], [3], [4] and [5]. Due to these facts, in this article the forecasting will be associated with the notion of prediction and will determine the future

Otilia Elena Dragomir is with Valahia University of Targoviste, Electrical Engineering Faculty, Automation, Computer Science and Electrical Engineering Department, 18 Unirii Av., Targoviste, Romania (email:drg otilia@yahoo.com).

Florin Dragomir is with Valahia University of Targoviste, Electrica Engineering Faculty, Automation, Computer Science and Electrical Engineering Department, 18 Unirii Av., Targoviste, Romania (e-mail: drg florin@yahoo.com).

Rafael Gouriveau is with Institut FEMTO-ST, CNRS - UFC / ENSMM UTBM, Département AS2M,24 rue Alain Savary, 25000 Besançon, France (e-mail: gouriveau@ens2m.fr)

Eugenia Mincă is with Valahia University of Targoviste, Electrica Engineering Faculty, Automation, Computer Science and Electrical Engineering Department, 18 Unirii Av., Targoviste, Romania (e-mail: minca@valahia.ro). state of the analyzed system the closest possible to the future real state of the system.

Different forecasting time horizons are employed in prediction approaches (e.g., day-ahead, hour-ahead) in relation with the application:

Short term load forecasting (STLF) [6] samples the information on an hourly (or half hourly) basis, or even a daily basis (for load peak prediction) so is defined as varying from a few minutes up to a few weeks ahead. This type of forecasting is important because the national grid requires DPcg values at any moment in the day. Traditionally, hourly forecasts with a lead time between one hour and seven days are required for the scheduling and control of power systems. Medium term load forecasting (MTLF) [7] covers the horizon from one month up to a few years ahead.

Long term load forecasting (LTLF) [7] considers load peaks and consumed energy, on a yearly basis, for several years ahead. For example, long-term forecasting is relevant for the planning of new electricity utilities, and inaccurate predictions have important financial costs. For this particular forecasting case is a need for accurate prediction.

Despite the many publications and models of load forecasting that have been developed in the last few decades, few amongst those have dealt with the medium term forecasting. From the perspective of the system operators and regulatory agencies, the medium term forecasting is a source of primary information for the safe and reliable operation of the system. For producers also, these ones are a basic tool for determining the optimal utilization of generators and power stations, as some facilities are more efficient than others.

This paper deals with this type of forecasting: medium term load forecasting. Precisely, it is used a modified neuro fuzzy network- ANFIS (Adaptive Neuro Fuzzy Inference System) to estimate the DPcg evolution for a medium time horizon. This artificial intelligence tool is applied on a data base obtain from an experimental photovoltaic amphitheatre of minimum dimension $(0.4 \mathrm{kV} / 10 \mathrm{~kW})$, located in the eastcentre region of Romania, more precisely in the city of Targoviste [8].

In this context, the paper is organized in two parts. First of all, the forecasting framework is delimited, starting with the definition, metrics, approaches and tools. In the second section a neuro- fuzzy network is used for medium term forecasting of DPcg. Precisely is proposed a new ANFIS 
architecture. The accuracy and the stability of the prediction error for the chosen forecasting horizon is illustrated and discussed.

Efforts improving the photovoltaic (PV) module performance in different climates have been increasing over the years. With new materials entering the market place, this task is gaining further in importance. Using standard test condition efficiency in the design stage is not enough appropriate. It is therefore imperative a true understanding of the reasons for varying performance before the design stage. Additionally, the accurate separation and quantification of different effects influencing the performance would be valuable asset to the PV community.

\section{FORECASTING IN POWER SYSTEMS}

\section{A. Forecasting concepts and measures}

There is no general agreement as to an appropriate and acceptable set of metrics that can be employed effectively to assess the performance of the prediction.

Firstly, it is very important the determination of error value (where the error is defined as the difference between real and estimated value of the system' $\mathrm{s}$ estimated parameter) [9]. Therefore, given the same error size corresponding to a certain magnitude of deviation, it is suitable to have a positive bias (named early prediction), rather than a negative one (fig $1 \mathrm{~b}$ ). Of course, one needs to define two different boundaries for the maximum acceptable late prediction and the maximum acceptable early one. Any prediction outside of it will be considered either a false positive or a false negative [10].

The performance measures will evolve with time as more data are available, and the measures are expected to improve over time. On the other hand, measurement of forecasting performance quality is highly dependent on how rigidly the criteria are specified, and are is largely dependent on userspecifications [11].

So a critical point at this level is what criterion can be used to measure the accuracy of the predicted results. Accuracy measures the closeness of the predicted value to the actual value. It is highest when the predicted value is the same as the actual value and decreases when the predicted value deviates from the actual value so the sensitivity will be very low when the predicted value deviates too much.

Another metric, often use in a prediction performance measure is the precision. The precision implies how close the predictions are bunched or clustered together and is a measure of the narrowness of un interval in which the system falls. Precision is defined on the basis of the variance of the predicted results for many experiments It is high if the predicted values are clustered together around the actual value and it is low if the predicted values are scattered over the output range (fig. $1 \mathrm{~b}$ ).
Confidence level of the forecasting indicates in percentage value, the degree of certitude of the future predicted value modes or of the estimation made. An average of the width of the confidence intervals of the prediction algorithm in the precision definition is often use because a narrower confidence interval gives higher precision (fig. 1 a).

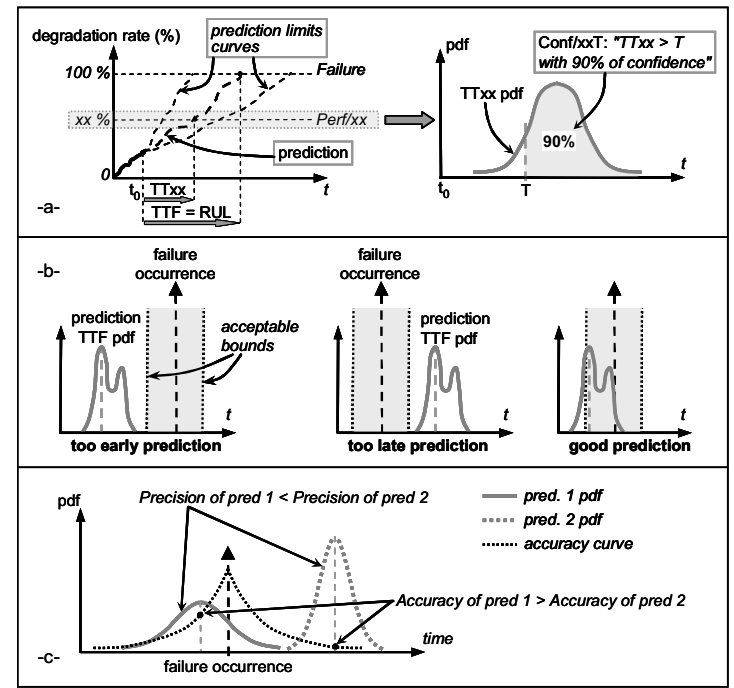

Fig. 1. Forecasting error metrics

\section{B. Forecasting approaches}

The literature has presented a great variety of approaches for electricity forecasting.

Contributions can be distinguished between statistical approaches and exponential smoothing approaches, between unvaried methods and methods with explanatory variables, between linear models and nonlinear models. Earlier papers have developed both single-equation models and multipleequation models with different equations for different hours of the day. The time dependence of hourly loads has been captured observation-driven models and parameter-driven models with unobserved components. Articles that deal with model based load forecasting include: [12], [13], [14], [15] and [16].

However, deregulated energy markets have presented new challenges to decision making, requiring more information which is forecasting dependent. Therefore, the corresponding development and maintenance efforts for dealing with hundreds of irregular data series, which need to be simultaneously forecasted for security and economical analyses, means that the parametric models are beyond practical consideration. The relationship between the electricity load and its exogenous factors is complex and nonlinear, making it quite difficult to represent using linear models, or even parametric nonlinear ones.

In recent years, papers have shown the potential of data driven forecasting approaches [17]. Data-driven approaches 
derive directly from routinely monitored system operating data. So, in many applications, measured input/output data is the major source for a deeper understanding of the system degradation behavior. These rely on the assumption that the statistical characteristics of data are relatively unchanged unless a malfunctioning even occurs.

The strength of data-driven techniques is their ability to transform high-dimensional noisy data into lower dimensional information for diagnostic/prognostic decisions. Artificial intelligence (AI) techniques have been increasingly applied to systems forecasting and have shown improved performances over conventional approaches.

In practice however, it isn't easy to apply AI techniques due to the lack of efficient procedures to obtain training data and specific knowledge. So far, most of the applications in the literature just use experimental data for model training. Thus, data-driven approaches are highly-dependent on the quantity and quality of system operational data.

\section{Forecasting tools}

Actually the systems are very complexes and the conditioning parameters that influence system functioning are significant. Often their actions induce nonlinearities in system modeling process because cannot be always precisely described. In these cases it is very difficult to determine any sort of model for prediction purposes.

Artificial Neural Networks (ANNs) are techniques use often for load forecasting. For short term forecasting horizons in particular, the neural networks have a good flexibility in capturing nonlinear interdependencies between the load and exogenous variables. However, the ANN models are complex and difficult to understand, and are often over-fitted. Indeed, their structure is sufficiently opaque that it is not clear why they should forecast as well as they do. As a result, the literature is still undecided as to their practical utility for electricity load forecasting. Articles that deal with ANN models, in particular for short term load forecasting horizon are: [18], [19], [20], [21], [22] and [23].

Despite all publicized successes, neural network based load forecasting models have been designed relying on timeconsuming, empirical, and suboptimal procedures. The potential for applying neural networks to short term load forecasting depends strongly on the extraction of appropriate input variables. This requirement has often been neglected, and many proposals for building the neural network input space still use (linear) correlation analysis. Input selection procedures with the capacity to extract high order statistical information from the input-output data must be employed to fully exploit the ANN mapping capability.

The advantages and the drawbacks of AI forecasting techniques, precisely of ANN, leaded us to neuro-fuzzy systems - NFs as reference tool for our approach of medium term energy balance forecasting.

Neuro- fuzzy systems are a combination of ANNs and fuzzy sets and represent a powerful tool to model systems behavior. The ANN is used to define the clustering in the solution space, which results in creation of fuzzy sets [24].

A particular architecture of neuro-fuzzy systems is represented by the adaptive neuro-fuzzy inference system (ANFIS) [25]. ANFIS is a Sugeno-type fuzzy inference system in which the parameters associated with specific membership functions are computed using either a back propagation gradient descent algorithm alone or in combination with a least squares method. It has been widely applied to random data sequences with highly irregular dynamics [26] e.g. forecasting non-periodic short-term stock prices [27].

The success of ANFIS is given by aspects like: the designated distributive inferences stored in the rule base, the effective learning algorithm for adapting the system's parameters or by the own learning ability to fit irregular or non-periodic time series. On the other hand, used in application alone to non-periodic short-term forecasting, ANFIS predictions make large residual errors due to high residual variance, consequently degrading prediction accuracy [28]. It is very difficult to interpret for a non expert the fuzzy rules generated by ANFIS because of the form of consequents (linear combination of inputs).

\section{DPCG FORECASTING USING ANFIS}

\section{A. Hypotheses}

Considering the bibliographical research made and the identified approaches, tools and forecasting metrics, in our approach we have decided to use ANFIS as forecasting tool for DPcg prediction on medium term horizon.

The ANFIS was applied on a data base obtain from an experimental photovoltaic amphitheatre of minimum dimension $(0.4 \mathrm{kV} / 10 \mathrm{~kW})$, located in the east-centre region of Romania, more precisely in the city of Targoviste.

In this context, the challenge consists in controlling the performance of the future state of the system for medium term. Precisely, we have to be able to ensure a certain level of error for a given prediction horizon (fig. 2).

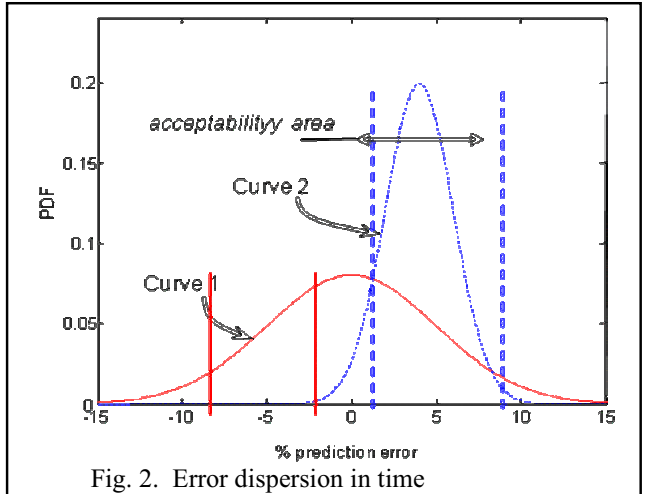

Fig. 2. Error dispersion in time 
Fig 2 represents our perception on error prediction control/ stabilization: although not centered at zero, the dispersion error of curve 2 suggests a prediction model more reliable than the curve 1 .

We consider the forecasting process as an aggregate function of past, present and future states. The third variable who appears in prediction function definition is the results of the necessity of taken into account of "known future" actions dues to precise moments in the week or in the year when we know that is scheduled a certain action. For example, it is expected a modification of consumers behaviors due summer, winter holydays or week-end time. In this case the prediction process will gain in accuracy because is an extra information that can be processed (Fig. 3).

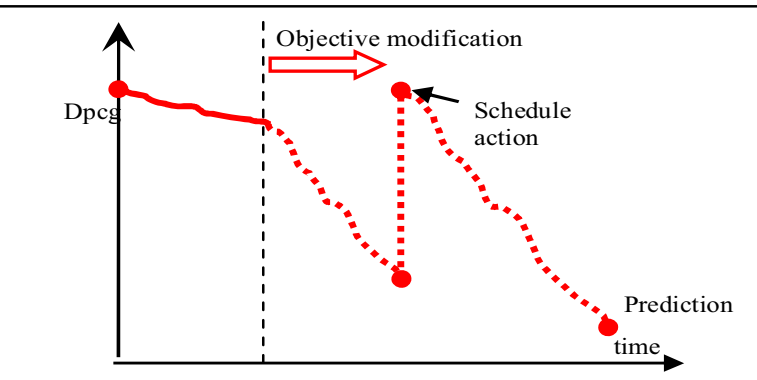

Fig. 3. Future states that influence prediction process

\section{B. Performance criteria}

The choice of error measures to help comparing forecasting methods has been much discussed. A major part of them have been summarized by [29]. Most authors consider that MAPE (mean absolute percent error) would be an adequate error measure if the loss function were linear (and linear in percentage, not in absolute error). However, recent studies and the experience of system operators indicates that the loss function in the load forecasting problem is clearly nonlinear, and that large errors may have disastrous consequences for a utility [30].

Because of manner of the penalization of large errors this, measures based on root mean squared error (RMSE) was suggested in. Also, it is generally recognized that error measures should be easy to understand and closely related to the needs of the decision-makers. Some papers reported that the utilities would rather evaluate forecasting systems by the absolute errors produced, and this suggests that mean absolute errors could be useful.

In any case, error measures are only intended as summaries for the error distribution. This distribution is usually expected to be normal white noise in a forecasting problem, but it will probably not be so in a complex problem like load forecasting. No single error measure could possibly be enough to summarize it. The shape of the distribution should be suggested. Keeping the total error low, therefore, means keeping the model simple.

It is well known that goodness-of-fit statistics are not enough to predict the actual performance of a method. The implications and consequences of the choices made in design, implementation and validation of neuro-fuzzy architecture are very important. Often appears problems of overfitting or overparametrization [31].

\section{Simulations results}

Considering the metrics described above, we have observed that "classical" ANFIS with two selected inputs (model 1 in our simulations), has satisfactory results for a short term prediction. For medium and long term, the obtained errors became bigger and bigger and this affects the forecasting in terms of accuracy and confidence (Fig. 4).

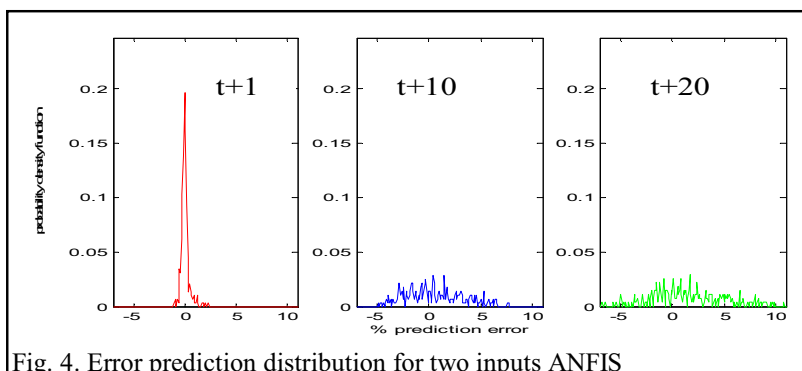

Fig. 4. Error prediction distribution for two inputs ANFIS

The RMSE grows from 0,4018 in case of $(t+1)$ at 3,8307 in case of $(\mathrm{t}+10)$. The MAPE penalizes the growth tendency of error in time and its values reaches 5,42 at $\mathrm{t}+10$ (table 1).

Table 1- RMSE and MAPE for model 1

\begin{tabular}{|c|l|l|l|}
\hline Variable & $\begin{array}{c}\text { Prediction } \\
\text { horizon }\end{array}$ & RMSE & MAPE \\
\hline \multirow{3}{*}{ Model 1 } & $\mathrm{T}+1$ & 0.4018 & 0.4618 \\
\cline { 2 - 4 } & $\mathrm{T}+10$ & 3.8307 & 5.4214 \\
\cline { 2 - 4 } & $\mathrm{T}+20$ & 0.4018 & 0.4618 \\
\hline
\end{tabular}

Thus, the performance metrics related to model 1 show that this model does not achieve a satisfactory compromise between short-term accuracy and long-term stability.

A first way to stabilize the prediction error is to link two ANFIS systems in series. We applied this approach in designing the second model for simulation (model 2): the first ANFIS system was set to make predictions at " $t+1$ " and was used "prev" times to make a prediction at " $t+$ prev". In this case, RMSE and MAPE metrics (fig. 5) show that prediction error seems to be stabilizing for a medium-term. 


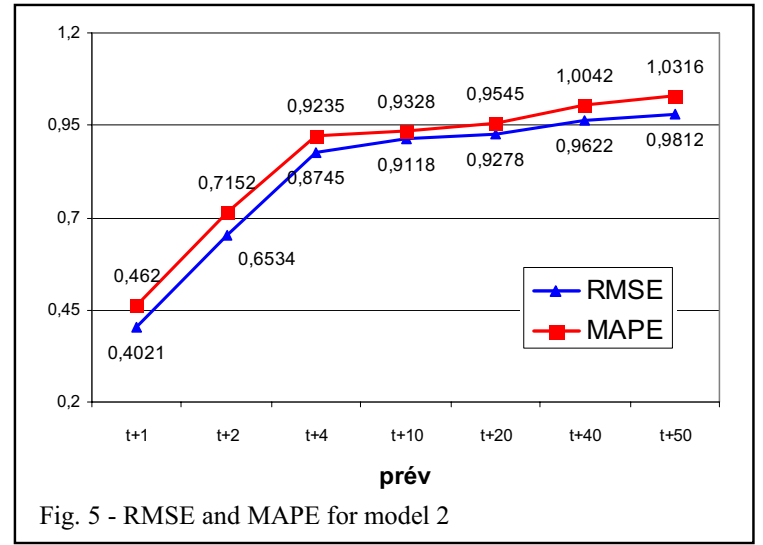

We have observed, with the error distribution (Fig. 6), that the accuracy of prediction improves also. The growth trend of the error, observed in the medium term in case of model 1 , is reduced.

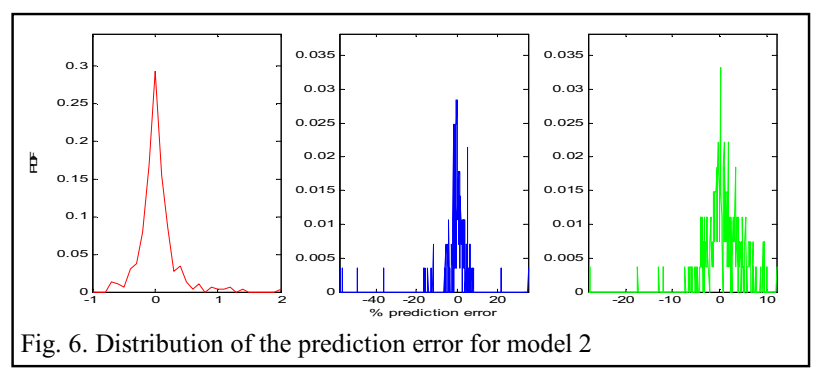

The standard deviation of the predictions on the one hand and the values of MAPE and RMSE on the other hand shows a more stable prediction process.

In order to obtain a better performance related to our objective, we have built a third model (model 3) considering the reasoning explained in fig. 3 .

The effect of taking into account the "future" for prediction, in the third case, in other words the modification of the mission profile due to some extern intervention has a good influence over the error (Fig. 7).

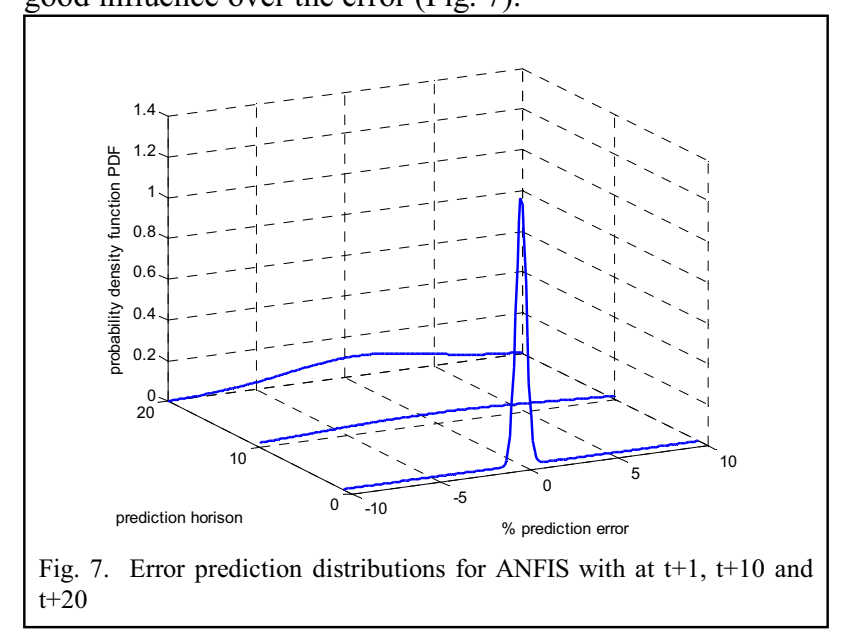

This proposition has helped the predictor to concentrate the distribution curves of the prediction error, particularly in the medium term case. The quality (stability) of the prediction model also tends to be independent of the prediction horizon.

\section{CONCLUSION AND WORK IN PROGRESS}

Controls of the performance prediction represent the premise of a good global forecasting performance. The existing approaches, methods, metrics and tools for load forecasting are discussed from different points of view. The identified problems and advantages create the context for proposing a new ANFIS architecture capable to ensure for the prediction stability in terms of errors magnitude for a medium term horizon. Also, the preplanned actions require the availability of assets under consideration for a specified time. The obtained confidence level at this point of time is a satisfactory one from the industrials point of view.

The work is still in progress and the developments are at present extended in three principal ways.

First, the definition of new loss functions capable to penalize the errors above an acceptable tolerance level.

Secondly, the investigation and the application of other tools as techniques for a global forecasting.

Finally, the amelioration of the forecasting system in terms of interpretability.

\section{REFERENCES}

[1] F. Dragomir, S.Iliescu, O.E. Dragomir and E.Minca, "PV grid connected system with fuzzy intelligent control”, Journal Computer Science and Control Systems, Vol.2, Nr.1,pg.97-101, 2009

[2] A. Rytter, Vibration Based Inspection of Civil Engineering Structures, PhD Thesis, 1993

[3] D.Lin, V. Makis, "Recursive filters for a partially observable system subject to random failure", Advances in Applied Probability, vol. 35, pp.207-227, 200)

[4] ISO 13381-1 (2004). Surveillance et diagnostic des machinesPronostic. Norme internationale- AFNOR

[5] O.E. Dragomir, R. Gouriveau, N. Zerhouni, F. Dragomir, "Framework for a distributed and hybrid prognostic system", in Proc. 4th IFAC Conference on Management and Control of Production and Logistics, 27-30 Sept., Sibiu, Romania, 2007

[6] H.S. Hippert, C.E. Pedreira and R.C. Souza, "Neural networks for short-term load forecasting: a review and evaluation", IEEE Transactions on Power Systems, vol.16, pp. 44-55, 2001

[7] J.Y.Desons, R.Ben Younes. Prévision long terme a la réponse d'un stockage de chaleur sensible dans le sol, Elsevier Science, 1997

[8] European Research Programm ICOP-DEMO 4080/98, "Building Integration of Solar Technology" (http://dcem.valahia.ro)

[9] G.Vachtsevanos, F.L. Lewis, M. Roen, A. Hess and R. Wu, Intelligent Fault Diagnosis and Prognosis for Engineering System, John Wiley and Sons Inc, USA, 2006

[10] K. Goebel, P. Bonissone, "Prognostic information fusion for constant load systems", in Proc. 7th annual Conference on Fusion, 2, 12471255,2005 
[11] P. Bonissone, K. Goebel, "When will it break? A Hybrid Soft Computing Model to Predict Time-to-break Margins in paper machine's", in Proc. SPIE 47th Annual Meeting, Int. Symp. Optical Science and Technology, 4787, 53-64, 2002

[12] J.R.Cancelo, A. Espasa, R. Grafe, "Forecasting from one day to one week for the Spanish system operator", International Journal of Forecasting, vol. 24, pp. 588-602, 2002

[13] J.M. Liu, R. Chen, L.M. Liu, J.L. Harris, “ A semiparametric time series approach in modeling hourly electricity loads", Journal of Forecasting, vol. 25, pp. 537-559, 2006

[14] L.J. Soares, M.C. Medeiros, "Modeling and forecasting short-term electricity load: a comparison of methods with an application to Brazilian data", International Journal of Forecasting, vol. 24, pp. 630-644, 2008

[15] J.W. Taylor, L.M. De Menezes, P.E. McSharry, "A comparison of univariate methods for forecasting electricity demand up to a day ahead", International Journal of Forecasting, vol. 22, pp. 1-16, 2006

[16] J.W.Taylor, R. Buizza, "Using weather ensemble predictions in electricity demand forecasting", International Journal of Forecasting, vol. 19, pp. 57-70, 2003

[17] O.E. Dragomir, R. Gouriveau, F. Dragomir, E. Minca, "Review of the prognosis problem in academicals and industrial area of interest", in Proc. European Control Conference 2009-ECC'09, 23-26 August Budapest, Hungary, 2009

[18] A.P. Alves da Silva, L.S. Moulin, "Confidence intervals for neural network based short term load forecasting", IEEE Transactions on Power Systems, vol. 15(4), pp. 1191-1196, 2000

[19] A. Khotanzad, R. Afkhami-Rohami, D. Maratukulam , "ANNSTLF Artificial Neural Network Short Term Load Forecaster - Generation Three", IEEE Transactions on Power Systems, vol. 13(4), pp. 1413-1422, 1998

[20] G. Darbellay, M. Slama, "Forecasting the short term demand for electricity: Do neural networks stand a better chance?", International Journal of Forecasting, vol. 16, pp. 71-83, 2000

[21] K. Metaxiotis, A. Kagiannas, D. Askounis, J. Psarras, “ Artificial intelligence in short term electric load forecasting: A state of the art survey for the researcher", Energy Conversion and Management, vol. 44, pp. 1525-1534, 2003

[22] A.J.R. Reis, A.P. Alves da Silva, "Feature Extraction Via MultiResolution Analysis for Short Term Load Forecasting", IEEE Transactions on Power Systems, vol. 20(1), pp. 189-198, 2005

[23] H.S. Hippert, D.W. Bunn, R.W. Souza , "Large neural networks for electricity load forecasting: Are they overfitted?", International Journal of Forecasting, vol. 21, pp. 425-434, 2005

[24] J.S.R Jang, C.T. Suni, E. Mizutani, Neuro-fuzzy and soft computing: a computational approach to learning and machine intelligence, Prentice Hal, New York, 1997

[25] J.S.R Jang, “ ANFIS: adaptive-network-based fuzzy inference systems”, IEEE Trans. Systems, Man, Cybern., vol. 23 (3), pp. 665 685., 1993

[26] J.S. Wang, “An efficient recurrent neuro-fuzzy system for identification and control of dynamic systems", IEEE Internat. Conf. Systems, Man, and Cybernetics, 2003

[27] L.H. Chiang, E. Russel, R. Braatz, Fault detection and diagnosis in industrial systems, Springer-Verlag, London, 2001

[28] C. Gourierou, ARCH Models and Financial Applications, Springer, NewYork, 1997

[29] J.G. Gooijer, R.J. Hyndman, “ 25 years of time series forecasting”, International Journal of Forecasting, vol. 22 , pp. 443- 473, 2006

[30] B.F. Hobbs, S. Jitprapaikulsarn, S. Konda, V. Chankong, K.A. Loparo, D.J. Maratukulam, " Analysis of the value for unit commitment of improved load forecasting", IEEE Trans. Power Systems, vol. 14 (4), pp. 1342-1348, 1999

[31] H. Steinherz, Carlos Eduardo Pedreira, Reinaldo Castro Souza, "Neural Networks for Short- term Load Forecasting: A Review and Evaluation”, IEEE Transactions on Power Systems, vol. 16(1), 2001 